This document is the accepted manuscript version of the following article:

Salces-Castellano, A., Patiño, J., Alvarez, N., Andújar, C., Arribas, P., Braojos-Ruiz, J. J., ... Emerson, B. C. (2020). Climate drives community-wide divergence within species over a limited spatial scale: evidence from an oceanic island. Ecology Letters, 23(2), 305-315. https://doi.org/10.1111/e1e.13433

\title{
Letter
}

\section{Climate drives community-wide divergence within species over a limited spatial scale: Evidence from an oceanic island}

Antonia Salces-Castellano ${ }^{1,2 \dagger}$, Jairo Patiño ${ }^{1,3+}$, Nadir Alvarez ${ }^{4}$, Carmelo Andújar ${ }^{1}$, Paula Arribas ${ }^{1}$, Juan José Braojos-Ruiz ${ }^{5}$, Marcelino del Arco-Aguilar³, Víctor García-Olivares ${ }^{1,2}$, Dirk Karger ${ }^{6}$, Heriberto López ${ }^{1}$, Ioanna Manolopoulou ${ }^{7}$, Pedro Oromí ${ }^{8}$, Antonio J. Pérez-Delgado ${ }^{1,2}$, William E. Peterman $^{9}$, Kenneth F. Rijsdijk ${ }^{10}$, \& Brent C. Emerson ${ }^{1 *}$

1. Island Ecology and Evolution Research Group, Institute of Natural Products and Agrobiology (IPNACSIC), C/Astrofísico Francisco Sánchez 3, La Laguna, Tenerife, Canary Islands, 38206, Spain

2. School of Doctoral and Postgraduate Studies, University of La Laguna, 38200, La Laguna, Tenerife, Canary Islands, Spain

3. Plant Conservation and Biogeography Group, Department of Botany, Ecology and Plant Physiology, University of La Laguna, C/ Astrofísico Francisco Sánchez, 38206 La Laguna, Tenerife, Canary Islands, Spain.

4. Natural History Museum of Geneva, 1 route de Malagnou, 1208 Geneva, Switzerland.

5. Tenerife Insular Water Council (CIATF), C/ Leoncio Rodríguez 2, 38003, Santa Cruz de Tenerife, Spain.

6. Swiss Federal Research Institute WSL, Züricherstrasse 111, 8903 Birmensdorf, Switzerland

7. Department of Statistical Science, University College London, London, United Kingdom.

8. Department of Animal Biology, Edaphology and Geology, University of Laguna, C/ Astrofísico Francisco Sánchez, 38206 La Laguna, Tenerife, Canary Islands, Spain.

9. School of Environmental and Natural Resources, The Ohio State University, Columbus, OH, USA.

10.Institute for Biodiversity and Ecosystem Dynamics, University of Amsterdam, Amsterdam, Netherlands.

+ These authors contributed equally

* Corresponding author: bemerson@ipna.csic.es, ph: +34 922256847

Key words: climate, topography, dispersal, gene flow, Quaternary, arthropod, beetle, speciation

Running title. Climate drives community-wide diversification

Author contributions. B.C.E. designed and coordinated the study. A.J.P.-D., H.L., P.O. and B.C.E did the field work. A.J.P.-D led sample sorting with input from H.L., A.S.-C. and P.O. H.L. and A.S.C generated the mtDNA sequence data. J.P. and V.G.-O. generated the ddRAD-seq data. D.K. generated the topoclimatic data, with input from J.P. A.S.-C. led the mtDNA analyses with input from 
B.C.E., J.P., I.M., P.A. and C.A. J.P. led ddRAD-seq data analyses, with input from B.C.E. and N.A. J.P. conducted ResistanceGA analyses, with input from W.E.P. M.J.d.A.-A., J.J.B.-J. and K.R. contributed local floristic, climate and geological expertise, respectively. B.C.E., A.S.-C. and J.P. wrote the manuscript, with contributions from all authors.

Data availability. Mitochondrial sequence data, filtered SNP data and topoclimate data are available from the Dryad Digital Repository https:doi.org.[to be made available upon acceptance]. Raw demultiplexed sequences for the full ddRAD-seq data set are available on NCBI SRA (BioProject Accession [to be made available upon acceptance].
Abstract word count: 152
Main text word count: 5000
Number of references: 65
Number of figures: 4
Number of tables: 0 


\begin{abstract}
Geographic isolation substantially contributes to species endemism on oceanic islands when speciation involves the colonisation of a new island. However, less is understood about the drivers of speciation within islands. What is lacking is a general understanding of the geographic scale of gene flow limitation within islands, and thus the spatial scale and drivers of geographical speciation within insular contexts. Using a community of beetle species, we show that when dispersal ability and climate tolerance are restricted, microclimatic variation over distances of only a few kilometres can maintain strong geographic isolation extending back several millions of years. Further to this, we demonstrate congruent diversification with gene flow across species, mediated by Quaternary climate oscillations that have facilitated a dynamic of isolation and secondary contact. The unprecedented scale of parallel species responses to a common environmental driver for evolutionary change has profound consequences for understanding past and future species responses to climate variation.
\end{abstract}




\section{INTRODUCTION}

Islands are often viewed as theatres for adaptive evolutionary change and speciation (Losos \& Ricklefs 2009), where non-adaptive paths to speciation are frequently given limited importance, or even ignored (e.g. Cabral et al. 2019). Recent models have sought to understand how geophysical island attributes influence insular biodiversity through the regulation of colonisation, speciation and extinction (e.g. Whittaker et al. 2008; Fernández-Palacios et al. 2016; Lim \& Marshall 2017). Although such models have sought to account for both long-term island ontogeny (e.g. Whittaker et al. 2008; Borregaard et al. 2017) and shorter-term surface area changes (e.g. Fernández-Palacios et al. 2016; Weigelt et al. 2016), insular species themselves have received much less attention (but see Rosindell \& Harmon 2013; Rominger et al. 2016; Cabral et al. 2019 for models incorporating species properties and biotic interactions). While much quantitative molecular data exists on insular speciation across archipelagos (e.g. Shaw \& Gillespie 2016), it is difficult to compare across such studies and generalise about the drivers of speciation within islands. This is because independent studies are idiosyncratic with regard to geographic sampling, and tend to be taxonomically biased toward diversified lineages. More importantly, such studies are largely focussed on describing patterns of speciation rather than the underlying processes that give rise to speciation. Despite these limitations, support has been found for a model where there is a significant correlation between the spatial scales of gene flow and speciation (Kisel \& Barraclough 2010). However, the importance and mechanistic basis of non-adaptive evolutionary pathways to speciation, where physical disruption of gene flow initiates divergence and ultimately promotes speciation, remains poorly understood.

Goodman et al. (2012) have noted that relatively rapid geological events within islands may be important drivers of divergence and speciation, and several studies implicate the fragmenting actions of volcanic activity (e.g. Vandergast et al. 2004) and flank collapses (e.g. Brown et al. 2006) as dispersal barriers. However, little attention has been focussed on the potential for local climate variation to act as a barrier to dispersal. Recently, García-Olivares et al. (2019) have proposed an 
insular topographic Quaternary diversification (ITQD) model, whereby genetic variation is structured by the geography of suitable climate. They used nuclear genomic data for a species complex of weevils (Laparocerus tessellatus) on the island of Gran Canaria (Canary Islands) to present evidence for geographic variation in humidity structuring genetic variation. Further to this, their results supported the influence of Quaternary climatic oscillations on contemporary patterns of genomic relatedness among individuals.

The results of García-Olivares et al. (2019) suggest a potentially important role for climate as a driver of population isolation and differentiation within islands, as landscape discontinuities for climate may similarly impact all species co-occurring in a given territory. Here we use laurel forest beetle species to test the hypothesis that fine-scale spatial variation in microclimate can disrupt gene flow and structure genetic variation in a concerted manner across species. Laurel forests occur in areas of high humidity, and are the ecosystem characterised by the highest levels of arthropod diversity within Macaronesia, of which many species are hydrophilic and restricted to this ecosystem (Fernández-Palacios et al. 2017). Both humidity and temperature are known to vary geographically within laurel forests (Braojos Ruiz 2015), providing the potential for geographic structuring of genetic variation within species. If landscape variation in humidity and temperature results in unfavourable areas for individual survival and/or establishment, then areas of favourable environmental conditions will be isolated if species are unable to disperse across unfavourable areas.

We focus our sampling within the laurel forest of the Anaga peninsula on the Canary Island of Tenerife, as the recent geological history of Anaga is one of relative stability. Anaga began to grow in the late Miocene, with the last major period of its polygenetic evolution about 4.2 Ma (million years ago) (Thirlwall et al. 2000), with relative quiescence from around $3.3 \mathrm{Ma}$ (Ancochea et al. 1990). The absence of younger volcanoclastics on Anaga indicate minimal impact from intense volcanic activity over the last several million years in the centre and south of the island (Carracedo 2011). Additionally, while most of the northern flank of Tenerife has suffered catastrophic flank collapses, some as recently as $150 \mathrm{Ka}$ (thousand years ago) (García-Olivares et al. 2017), only one 
such flank collapse is known for the Anaga Peninsula, estimated to have occurred 4.1 - 4.7 Ma (Walter et al. 2005). Thus, the Anaga peninsula represents a relatively geologically stable region, where millennial-scale erosional activity and climate variation have been more consequential than catastrophic geological events, at least over the last $3 \mathrm{Ma}$. This provides a near ideal template within which to assess how landscape discontinuities for suitable environmental conditions impact upon spatial patterns of genetic relatedness within species.

We first generated six climatic and topographical layers that are expected to have some functional relationship to water stress or geographical isolation across the dorsal ridge that runs eastwest along the Anaga peninsula (Fig. 1), at a resolution of $90 \mathrm{~m}^{2}$. Within this area we then used an intensive sampling protocol (Emerson et al. 2017) to sample Coleoptera from ten 50m x 50m plots within the laurel forest of the peninsula, which were then classified to presumed biological species (PBS) using a modification of the protocol of Emerson et al. (2017). Mitochondrial DNA was used to generate estimates of population genetic structure among sampling sites within each PBS, and these individual estimates were then summarised as pairwise dissimilarity among sampling sites across all PBS. For comparative purposes, two pairwise beta diversity matrices were also calculated, one using PBS distribution data, and one using individual haplotypes. The five community-level dissimilarity matrices were then used to assess patterns of geographic structure and to test between hypotheses of isolation by distance, or isolation by unfavourable areas of higher water stress that limit gene flow in species with high humidity requirements. We then tested species-specific predictions derived from these results using genomic datasets obtained from double-digest restriction siteassociated DNA sequencing (ddRAD-seq) for 16 PBS.

\author{
MATERIALS AND METHODS
}

Sampling, specimen sorting into parataxonomic units and mtDNA sequencing 
Ten sampling plots of 50m x 50m were established within the laurel forest of the Anaga peninsula, on the island of Tenerife in the Canary Islands (Fig. 1, see Appendix S1 for further details). A standardised sampling protocol (Emerson et al. 2017) was applied to each plot, with sampling undertaken between Nov 2012 and April 2013. Specimen sorting into parataxonomic units (PUs) was conducted using the protocol of Emerson et al. (2017), with minor modifications (see Appendix S1). Within each plot, four individuals from each sampled PU (less if fewer than four were sampled) were sequenced for a 824 bp region of the mtDNA COI gene (see Appendix S1 for details).

\section{Delimitation of presumed biological species and taxonomic assignment}

We used a modification of Emerson et al. (2017) for the inference of PBS (see Appendix S1 for details). Final taxonomic assignment for PBS was made using the taxonomic literature and a reference collection of Canary Island beetles maintained by one of us (PO). For taxonomically complex groups for which a molecular phylogeny is available (e.g. Cryptorhynchinae (Stüben \& Astrin 2010), Laparocerus (Machado et al. 2017)), final taxonomic assignment was aided by phylogenetic assignment after sequencing several individuals of a PBS for a corresponding gene region.

\section{Bayesian inference of phylogeographic clusters}

We used the $\mathrm{R}$ package BPEC (Bayesian Phylogeographic and Ecological Clustering) (Manolopoulou et al. 2011) to identify genetically distinct geographical population clusters within each PBS. The program used a fully model-based framework to generate posterior probabilities for phylogeographic groupings, combining a parsimony-based evolutionary model for genealogical relationships among DNA sequences, together with a geographical model representing dispersal events consistent with geographically coherent clusters of related sequences. The following parameters were chosen to achieve convergence: (i) maximum number of migrations (maxMig = 5), (ii) parsimony relaxation parameter $(\mathrm{ds}=0$ ), and (iii) number of iterations (iter $=10,000,000)$. 
For each PBS a MCMC sampler was run to obtain phylogeographic clusters. Results for each PBS were then summarised within a pairwise matrix of locations where: $1=$ both locations were sampled for the same phylogroup, $0=$ each location was sampled for a different phylogroup, NA = one or both locations were not sampled. Results across all matrices were integrated to create a single matrix where each cell represents the proportion of PBS sampled from a pair of locations that belong to the same phylogroup.

\section{PBS and intraspecific mtDNA beta diversity and dissimilarity}

A pairwise beta diversity matrix, including beta components [i.e. $\beta_{\text {Sorensen, }} \beta_{\text {Simpson }}$ and $\beta_{\text {nestedness }}$ ] were calculated among all plots at the level of PBS using the R package betapart (Baselga \& Orme 2012). Pairwise dissimilarity of assemblages among sites was also assessed at the intraspecific level. First, we generated a pairwise beta diversity matrix at the haplotype level using the same approach as that for PBS, but in this case based on the presence/absence of haplotypes across all plots (Baselga et al. 2013). In this analysis all haplotypes from all PBS are used in a single analysis. Second, we assessed dissimilarity at the intraspecific level using (i) $\mathrm{G}_{\mathrm{ST}}$ to partition haplotype variation within PBS and (ii) $\mathrm{N}_{\mathrm{ST}}$ to also account for haplotype relatedness. For each PBS, total and pairwise $\mathrm{G}_{\mathrm{ST}}$ and $\mathrm{N}_{\mathrm{ST}}$ among plots were estimated using SPAGeDi (Hardy \& Vekemans 2002), and pairwise values were then used to calculate average $\mathrm{G}_{\mathrm{ST}}$ and $\mathrm{N}_{\mathrm{ST}}$ for all pairs of plots using all PBS, providing novel pairwise dissimilarity matrices at the intraspecific level.

\section{Hierarchical cluster analyses and homogeneity analyses}

The five dissimilarity matrices described above (PBS, phylogroup, $\mathrm{G}_{\mathrm{ST}}, \mathrm{N}_{\mathrm{ST}}$, and haplotype) were used to perform hierarchical cluster analysis using Ward's agglomerative method (Murtagh \& Legendre 2014). To identify the more strongly differentiated and internally homogenous groups of plots based on dissimilarity matrices, homogeneity analyses were performed to assess the increase in the average within-group pairwise similarity across the groups defined by the corresponding 
hierarchical classification (Bedward et al. 1992). Permutational ANOVAs (Permanova) were conducted to test for significant differences across the defined groups of plots for each of the five dissimilarity matrices.

\section{MtDNA network analyses and estimation of divergence times}

MtDNA haplotype networks for 15 PBS and a sister PBS pair selected for ddRAD-seq (see next section) were constructed in SplitsTree v.4.14.6 (Huson \& Bryant 2006), using the NeighborNet method with K2P distances. Divergence times were estimated with BEAST v.2.5.0 (Bouckaert et al. 2014), applying: (i) optimal models for each alignment inferred with jModeltest2 (Darriba et al. 2012) (Table S1); (ii) a strict clock, which is frequently used in analyses of sequence data sampled at the intraspecific level (Ho \& Duchêne 2014), for which the use of the relaxed molecular clock is not appropriate (Hein et al. 2005); (iii) a constant size coalescent prior, and; (iv), a uniform prior distribution representing the $95 \%$ confidence interval around a beetle specific rate estimate of $0.0177( \pm 0.0038)$ for the mtDNA COI gene (Papadopoulou et al. 2010). MCMC analyses were run for $100,000,000$ steps, sampling every 1,000 steps, with the first $10 \%$ discarded as burnin, using values of effective samples size (ESS) greater than 200 as a minimum for acceptance.

\section{Double-digest restriction site-associated DNA sequencing and de novo assembly}

Based on inferences from community-level analyses, 15 PBS and a sister PBS pair were selected for double-digest restriction site-associated DNA sequencing (ddRAD-seq). ddRAD-seq libraries were prepared following the protocol of García-Olivares et al. (2019) with some minor modifications (see results). ddRAD-seq libraries for each PBS were processed using the iPyrad v0.7.19 pipeline (http://ipyrad.readthedocs.io/). De novo assembly details can be found in Appendix S1.

\section{Genomic population structure and differentiation}


To explore population structure within each of the 16 ddRAD-seq datasets, we first constructed phylogenetic networks, using the NeighborNet algorithm implemented in SplitsTree 4 (Huson \& Bryant 2006). Principal component analyses (PCA) were performed with the R package adegenet v. 2.1.1 (Jombart \& Ahmed 2011). To explore population genetic structure across sampling sites, we used a model-based clustering analysis that estimates individual ancestry coefficients representing the proportions of an individual genome that originate from multiple ancestral gene pools. Calculations were implemented in the program sNMF based on sparse non-negative matrix factorization and least-squares optimization (Frichot et al. 2014). Ancestry coefficients estimated using the sNMF method have been shown to produce results that are comparable to other widely used programs such as ADMIXTURE and STRUCTURE, but have the advantage of estimating homozygote and heterozygote frequencies and avoiding Hardy-Weinberg equilibrium assumptions (Frichot et al. 2014). We calculated ancestry coefficients for 1-10 ancestral populations (K) using 10 replicates for each $\mathrm{K}$. We used a cross-entropy criterion, based on the prediction of masked genotypes to evaluate the error of ancestry estimation, to identify optimal values for K. The sNMF method was implemented in the R package LEA (Frichot et al. 2015).

\section{Climate modelling and environmental layers}

We modelled mean annual temperature (TEMP) and mean annual precipitation (PREC) using geographically weighted regression (GWR) with station data from the Spanish Agency of Meteorology (www.aemet.es). We used 152 stations and 217 stations for temperature and precipitation, respectively. A digital elevation model (DEM) at a resolution of $\sim 90 \mathrm{~m}^{2}$ resolution was derived from CGIAR-CSI SRTM v4.1 and ASTER GDEM v2 data products (Robinson et al. 2014). For temperature we calculated a GWR with elevation (ELEV) of the DEM as a predictor variable and a Gaussian kernel. For precipitation we also used a GWR with a Gaussian kernel, using the windward / leeward index (Böhner \& Antonic 2008; Karger et al. 2017), and elevation as predictors. We additionally applied a residual interpolation using the station data to remove uncertainties in 
temperature and precipitation not captured by the GWR. The residuals were then interpolated using a multilevel B-spline interpolation with fourteen error levels. The resulting layers were then added to the GWR predictions in order to obtain the final temperature and precipitation layers. For topographic wetness (WET) we used the SAGA wetness index (Böhner \& Antonic 2008) in SAGA GIS Vers. 6.0.0 (Conrad et al. 2015), in order to describe the tendency of a cell to accumulate water. Finally, from the ELEV layer in SAGA QGIS, two additional topographic indices of position (POS) and ruggedness (RUG) were estimated.

\section{Landscape resistance analyses}

We used the R package ResistanceGA v. 4.0 (Peterman 2018) to assess the effects of the landscape factors described above (TEMP, PREC, ELEV, WET, POS, RUG) on connectivity among sampling plots. Using a genetic algorithm and mixed effects models fit based on the maximum-likelihood population effects parameterization (Clarke et al. 2002), this method optimizes resistance surface values such that effective distances between sample locations across the landscape best fit the observed genetic differentiation or community dissimilarity between locations. ResistanceGA is unique among available approaches for landscape resistance modelling in that is a true optimization of landscape resistance values, avoiding subjectivity in assigning resistance values (Peterman et al. 2014; Peterman 2018).

We assessed the effects of the different landscape factors on multiple different pairwise distance measures. These distance measures were estimated at the level of sampling sites (i.e. community-level data) or individuals, as follows: (i) for the community-level matrices, we used the five dissimilarity matrices described above (i.e. PBS, phylogroup, $\mathrm{G}_{\mathrm{ST}}, \mathrm{N}_{\mathrm{ST}}$, and haplotype); and (ii) for individuals within each of the 16 ddRAD-seq data sets, we followed the approach proposed by Petkova et al. (2015). Effective distances between sample sites were calculated as the random-walk commute time (van Etten 2017), which is a multi-path distance measure equivalent to resistance distance (Kivimäki et al. 2014), as calculated by CIRCUITSCAPE (McRae et al. 2008; McRae \& 
Shah 2009). We used the "all_comb" function in ResistanceGA to optimize all single- and multiplesurface combinations of resistance surfaces (up to three surfaces within a model). For multiplesurface optimization, resistance surfaces were combined during optimization to create novel composite resistance surfaces. We used AICc to assess support for optimized resistance surfaces, and fitted mixed effects models with a $\triangle \mathrm{AICc}<2$ were deemed to be equally supported.

\section{RESULTS}

\section{Sampling and delimitation of presumed biological species}

A total of 23,180 Coleoptera specimens were sampled across the 10 plots, from which a total of 2,181 individuals were selected for DNA sequencing based on initial assignment to PUs, of which 1,919 were successfully amplified and sequenced. Two PUs, corresponding to the taxonomic species Tarphius canariensis and T. simplex were removed from subsequent analysis as they did not segregate based on mtDNA, consistent with previous suggestions of incomplete lineage sorting within this group (Emerson et al. 2000), or hybridisation. A total of 154 PBS were identified, of which 146 are identified as native or endemic to the Canary Islands, yielding a total of $1786 \mathrm{mtDNA}$ sequences corresponding to 950 different haplotypes.

\section{Community-level geographic structuring of genetic variation within species}

A consistent community-level signal for the structuring of intraspecific genetic variation into two regions was observed, hereafter referred to as East and West (Fig. 2). This contrasts with the pattern observed when dissimilarity was measured at the level of PBS. The intraspecific signature was strongest when phylogeographic groupings within PBS were considered, with the Permanova analysis revealing that $78 \%$ of dissimilarity among sampling plots was explained by phylogeographic differences between East and West. A more limited signal was recovered when intraspecific genetic variation was summarised as $\mathrm{N}_{\mathrm{ST}}$ and $\mathrm{G}_{\mathrm{ST}}$ (Permanova $\mathrm{R}^{2}=0.44$ an 0.38 respectively), while 
dissimilarity of haplotypes between East and West only explained $11 \%$ of the variation among sampling plots. Resistance landscape analyses of the six topoclimatic variables (Fig. 2c) revealed that patterns of community dissimilarity are best explained as a function of geographic distance when intraspecific variation is summarised with $\mathrm{N}_{\mathrm{ST}}$ and $\mathrm{G}_{\mathrm{ST}}$. When considering all haplotypes across all PBS, both temperature and geographic distance best explained variation in community similarity. When considering dissimilarity in PBS composition, both topographic wetness and geographic distance best explained dissimilarity among communities. However, when intraspecific variation was partitioned into phylogroups, landscape variation in mean annual precipitation alone followed by elevation best explained community dissimilarity.

\section{Sequencing and de novo assembly of ddRAD-seq data}

Seven PBS and a sister PBS pair were chosen to represent East-West structuring of mtDNA variation consistent with community-level patterns detected with the above-described analyses of mtDNA variation. Selected PBS presented East-West structure based on BPEC analyses together with significantly positive values for both $\mathrm{G}_{\mathrm{ST}}$ and $\mathrm{N}_{\mathrm{ST}}$. The sister PBS pair comprised two previously described mtDNA lineages (Schütte \& Stüben 2015) that assign unambiguously to Acalles globulipennis, with a divergence marginally exceeding our conservative $8 \%$ Kimura twoparameter threshold between species (see Methods and Appendix S1). Nuclear genomic data for these two PBS revealed congruent differentiation, but also evidence for incomplete reproductive isolation (see Results). For simplicity, we hereafter refer to all individuals of A. globulipennis as one PBS, while recognising it to be more consistent with a species complex.

A total of 235 individuals were selected for analysis, for an average of 14.7 $\pm 5.4(9-26)$ individuals per PBS (Table S2). A minimum level of DNA sample concentration $(>0.5 \mathrm{ng} / \mu \mathrm{l}) \mathrm{was}$ achieved for all samples, with an average concentration of $22.8 \pm 4.0 \mathrm{ng} / \mu 1$. A total of four libraries were prepared, randomly selecting four PBS for each library and including a randomly-selected replicate per PBS and a blank control per library. On the basis of fragment spectrums measured for 
each of the 16 PBS prior to pooling using a Fragment Analyzer (Advanced Analytical), libraries were then size selected between 225 bp and 275 bp using the Pippin Prep electrophoresis platform (Sage Science) and fragment spectrums for each pool were then verified with a Fragment Analyzer. All libraries were pooled and sequenced on four lanes of Illumina HiSeq2500 using the single-end (100 bp) protocol at the Lausanne Genomic Technologies Facility. Twenty-three samples did not sequence well and were removed from further analyses. The 16 ddRAD-seq libraries yielded an average of 44,685,100 raw reads (S.D. $=7,104,526)$. Depending on the PBS, we recovered between 2,775 and 34,339 loci, including from 2,663 to 33,835 unlinked biallelic SNPs, with between 0.9 to $7.19 \%$ missing data (Table S2).

\section{Species-specific testing of predictions from community-level inference}

Among the eight PBS with strong regional structuring of mtDNA variation, estimates for the onset of regional divergence ranged from $0.5 \mathrm{Ma}$ (Rhopalomesites persimilis, 95\% HPD: 0.2-0.8 Ma) to 4.6 Ma (Acalles globulipennis, 95\% HPD: 2.5-7 Ma) (Table S1). For all PBS, minimum spanning networks, principal component analyses and model-based ancestry estimation revealed three consistent features: (i) East-West structuring of nuclear genetic variation; (ii) evidence for ancestral populations consistent with East and West; and (iii) signatures of localised admixture among populations, frequently involving sites five and six, but in some cases extending to adjacent sites, with the exception of a single PBS not sampled at sites five and six (Fig. 3 and Fig. S1). Landscape resistance analyses revealed that patterns of genomic relatedness among individuals within all eight PBS are best explained by one or more climatic or topographical variables (Fig. S1 and Table S3). Geographic distance did not feature in the set of best supported models for any of the eight PBS.

Among the eight PBS presenting no evidence for regional structuring of mtDNA variation, three presented only limited signatures for geographic structure of nuclear genetic variation, with individual relatedness best explained by geographic distance, or the null model (Fig. S1). However, for the remaining five PBS, ddRAD-seq data revealed the same three features of (i) East-West 
separation, (ii) ancestral assignment to the East and West populations, and (iii) admixture centred around sites five and six. Additionally, genomic relatedness among individuals for all five PBS is best explained by climatic or topographical variables (Fig. S1). Maximum age estimates for the onset of regional divergence within these five PBS ranged from 0.1 Ma (95\% HPD: 0-0.2 Ma) to 0.9 Ma (95\% HPD: 0.4-1.4 Ma) (Table S1).

\section{DISCUSSION}

Strong support was found for community-level structuring of genetic variation between East and West when considering phylogeographic structure within PBS, with landscape variation for elevation and precipitation best explaining this structure (Fig. 2). The lack of similarly high support from other measures of intraspecific dissimilarity can be reconciled with the differing impact of unstructured PBS among the different analyses. Information content for the phylogeographic analysis is restricted to the 36 PBS that presented more than one phylogroup, while all PBS contributed to dissimilarity measures derived from $\mathrm{N}_{\mathrm{ST}}, \mathrm{G}_{\mathrm{ST}}$ and haplotypes. Thus, for these three measures it is plausible that regional signatures of East-West differentiation are confounded by alternative signatures, such as isolation by distance, present in other PBS.

The phylogeographic data support a general model where individual relatedness is described by topoclimatic variation, with the predominant structure being western and eastern populations. In total, eastern and western populations were inferred with nuclear genetic data for 13 of the 16 PBS analysed, of which five presented no regional mtDNA structure. Landscape variation for climate and topography explained geographic patterns of genomic relatedness within all 13. Twelve of these PBS presented signatures of admixture, which were concentrated around sites 5 and 6 for 11 PBS (Fig. 4). This is strong evidence for a shared dynamic within a community-level model, the explanation of which requires an understanding of the geological history of the Anaga peninsula itself. 
Instability of the northern flank of Anaga, between sites 1 and 6 (Fig. 1), led to partial seaward collapse, estimated between 4.1 - 4.7 Ma (Walter et al. 2005) of approximately $15 \mathrm{~km}^{2}$ of surface area (García-Olivares et al. 2017). A more than 4 million-year old topographic footprint of the Anaga mega-landslide is visible today as steep northern slopes and an approximately $115 \mathrm{~m}$ drop in elevation between sites 4 and 5 (Fig. 4). This saddle represents the lowest crestal elevation across the sampling area, and is likely to have been a consistent topographic feature of the region since the landslide happened (Fig. S2). Elevation itself is a proxy for an important source of moisture, not included in our model due to its local and idiosyncratic nature. A thermal inversion layer varies in elevation throughout the year, regulating the formation, intensity and frequency of stratocumulus clouds (Sperling et al. 2004). More intense and frequent cloud formation occurs at lower elevations (700900 metres above current sea level; macsl) during the regionally dryer summer months (Marzol 2008). With crest elevation dropping to as low as 675 macsl, the region between sites 4 and 5 falls below the cloud formation that maintains a humid environment from site 4 to the east and from site 5 to the west through condensation (del Arco Aguilar et al. 2006; Braojos Ruiz 2015). This limited water capture from condensation further contributes to water stress within the generally dryer and warmer climate of the saddle region (Fig. S3).

While the geographic distance between sites 4 and 5 (Fig. 1) measures only $3.7 \mathrm{~km}$, our data clearly reveal that the dryer environmental conditions spanning this distance have been consequential for a large number of beetle species over the last several million years. Results are consistent with a model of geographic isolation by niche conservatism of climatic tolerance (Wiens \& Graham 2005), in particular to humid conditions within the laurel forest, and facilitated by limited dispersal ability (flightlessness) for most species. While it is assumed that laurel forest beetle species are typically limited to this ecosystem (e.g. Fernández-Palacios et al. 2017), fundamental species distribution data is lacking. Using the limited species presence records that have been extrapolated from the literature, we applied the maximum entropy species distribution modelling approach (Maxent, Phillips et al. 2006), providing results consistent with laurel forest affiliations for 13 of the 15 PBS (for details on 
methods and results, see Appendix S2). Two simple and contrasting mechanistic explanations can be put forward to explain the onset of East and West isolation within species, either: (i) colonisation from one region to the other by chance dispersal, or (ii) range vicariance driven by periods of suboptimal climate impacting species ranges (Fig. S4). Vicariance caused by the mega-landslide itself is a likely explanation for the early Pliocene divergence estimated within Acalles globulipennis (Table S1). However, all remaining PBS have divergence time estimates within the Quaternary, where both mechanisms may plausibly explain the origin of isolation. For all PBS, irrespective of the mechanism, divergence must also be reconciled with repeated inferences of secondary contact, concentrated around sites 5 and 6 . In this context, cyclical altitudinal shifts in favourable environmental conditions are an expected consequence as Quaternary insular climate transitioned between glacial and interglacial conditions (Fernández-Palacios et al. 2016). The repeated evidence for coincident isolation and secondary contact presented here, together with mtDNA divergences ranging over the full extent of the Quaternary (Table S1), indicate a dynamic of isolation and secondary contact extending over multiple cycles of Quaternary climate oscillation. Recent climate models applied to laurel forest bryophytes (Patiño et al. 2016) predict that globally warmer interglacial conditions should result in higher water stress at lower elevations, and upslope shifts in range limits. Under such a model, regional isolation of hydrophilic beetles should be greatest during interglacial periods, such as now, with higher connectivity during cooler glacial conditions. However, an alternative model predicts reduced water stress at lower laurel forest elevations as climate transitions from glacial to interglacial conditions (Sperling et al. 2004), and it remains unclear which model best explains the spatiotemporal dynamics of the laurel forest. We suggest that these competing models, and their contrasting demographic predictions, could be directly tested with more detailed geographic sampling and genomic analysis of regionally structured species such as those presented here.

The importance of Quaternary climate oscillations for biodiversity distribution patterns among islands has recently been demonstrated (Rijsdijk et al. 2014; Weigelt et al. 2016). Our results extend this influence to within island diversification, revealing that subtle environmental 
discontinuity over a limited geographic distance can structure genomic variation across multiple species. Although we do not suggest that population divergence will inevitably lead to speciation in all species, the potential for eventual speciation is demonstrated by A. globulipennis, where divergence between East and West populations is consistent with morphologically cryptic species (Hendrich et al. 2015; see Appendix S1), and exceeds divergences between related species within the subfamily Cryptorhynchini (Stüben \& Astrin 2010). This strong and unprecedented support for a topoclimate-mediated, islands within islands model for speciation (García-Olivares et al. 2019) has important consequences for island biogeography, where it has previously been suggested that dispersal limitation and landscape variation may promote isolation and speciation within islands (Goodman et al. 2012), even at relatively small geographic scales (Vandergast et al. 2004). Topoclimatic mosaics should have been subjected to greater temporal stability during the Tertiary, enhancing isolation effects and the potential for speciation by reducing periodic opportunity for secondary contact. Thus, for taxa characterised by limitations to both dispersal ability and climatic tolerance, environmental discontinuities across topographically complex landscapes are likely to be a persistent driver of speciation throughout island ontogeny. More generally, our results suggest that topoclimatically variable continental landscapes may be important arenas for speciation, particularly when such landscapes were characterised by relative geological stability during the Tertiary, but were buffered from defaunation during Quaternary climatic oscillations. Finally, our results further highlight the sensitivity of arthropod species range limits to climate change (Robinet \& Roques 2010). We suggest that when species dispersal ability and environmental tolerance are restricted, demographic impacts of climate change will be stronger, which may offer some explanation for recently reported variation in climate-related abundance declines for tropical arthropods (Lister \& Garcia 2018).

Isolated populations represent the incipient stages of speciation, and are thus of fundamental importance for understanding speciation (Mayr 1942). We have revealed repeated and coincident geographic isolation and genomic divergence within species across a spatially limited environmental 
barrier, within a dynamic of divergence with gene flow. Divergence times within species range from less than $100 \mathrm{Ka}$ to more than $4 \mathrm{Ma}$, providing different temporal snapshots of divergence along the speciation continuum within the same geographic setting. Our results highlight not only the importance of integrating ecological and evolutionary data to reveal process-based explanations for the generation and spatial structure of biodiversity, but also the fine spatial scale within which such processes can play out. 


\section{ACKNOWLEDGEMENTS}

This research was supported by Spanish MINECO grants CGL2013-42589-P and CGL2017-85718P, co-financed by FEDER and awarded to B.C.E, and by the ERA-Net Net-Biome research framework, financed through Canary Island Government ACIISI grants SE-12/04 and SE-12/02, cofinanced by FEDER and awarded to P.O. and B.C.E respectively. A.S.-C. was funded by the Ministerio de Educación y Formación Profesional through the FPU PhD fellowship (FPU014/02948). J.P. was funded by the MINECO through the Juan de la Cierva Program - Incorporation (IJCI-201419691) and Ramón y Cajal Program (RYC-2016-20506), and Marie Sklodowska-Curie COFUND, Researchers' Night and Individual Fellowships Global (MSCA grant agreement No 747238, 'UNISLAND'). The authors wish to acknowledge the contribution of the Teide High-Performance Computing facility (TeideHPC) provided by the Instituto Tecnológico y de Energías Renovables (ITER), S.A, and to thank the following for assistance with field work and sample sorting: Rienk Apperloo, Manuel Arechavaleta, Salvador de la Cruz, Nuria Macías-Hernández, Benito Pérez Vispo, Sara Ravagni, Isa Sancibrián Span, and Nieves Zurita. We thank two anonymous reviewers for their insightful comments and useful suggestions. 


\section{REFERENCES}

Ancochea, E., Fúster, J.M., Ibarrola, E., Cendrero, A., Hernan, F., Cantagrel, J.M. et al. (1990). Volcanic evolution of the island of Tenerife (Canary Islands) in the light of new K-Ar data. Journal of Volcanology and Geothermal Research, 44, 231-249.

Baselga, A., Fujisawa, T., Crampton-Platt, A., Bergsten, J., Foster, P.G., Monaghan, M.T. et al. (2013). Whole-community DNA barcoding reveals a spatio-temporal continuum of biodiversity at species and genetic levels. Nature Communications, 4.

Baselga, A. \& Orme, C.D.L. (2012). betapart: an R package for the study of beta diversity. Methods in Ecology and Evolution, 3, 808-812.

Bedward, M., Keith, D.A. \& Pressey, R.L. (1992). Homogeneity analysis: Assessing the utility of classifications and maps of natural resources. Austral Ecology, 17, 133-139.

Böhner, J. \& Antonic, O. (2008). Land-surface parameters specific to topoclimatology. In: Geomorphometry: Concepts, Software, Applications. (eds. Hengl, T \& Reuter, HI). Elsevier, pp. 195-226.

Borregaard, M.K., Amorim, I.R., Borges, P.A.V., Cabral, J.S., Fernández-Palacios, J.M., Field, R. et al. (2017). Oceanic island biogeography through the lens of the general dynamic model: assessment and prospect. Biological Reviews, 92, 830-853.

Bouckaert, R., Heled, J., Kühnert, D., Vaughan, T., Wu, C.-H., Xie, D. et al. (2014). BEAST 2: a software platform for Bayesian evolutionary analysis. PLoS Computational Biology, 10, e1003537.

Braojos Ruiz, J.J. (2015). La nube, el pino y la otra lluvia. Una metodología para evaluar el potencial de captación de aqua de niebla y su aprovechamiento natural o artificial. Consejo Insular de Aguas de Tenerife, Santa Cruz de Tenerife.

Brown, R.P., Hoskisson, P.A., Welton, J.H. \& Báez, M. (2006). Geological history and within-island diversity: a debris avalanche and the Tenerife lizard Gallotia galloti. Molecular Ecology, 15, 3631-3640.

Cabral, J.S., Wiegand, K. \& Kreft, H. (2019). Interractions between ecological, evolutionary, and environmental processes unveil complex dynamics of insular plant diversity. Journal of Biogeography, 46, 1582-1597.

Carracedo, J.C. (2011). Geología de Canarias I. Origen, evolución, edad y volcanismo. Editorial Rueda, Madrid.

Clarke, R.T., Rothery, P. \& Raybould, A.F. (2002). Confidence limits for regression relationships between distance matrices: Estimating gene flow with distance. Journal of Agriculture, Biological , and Environmental Statistics, 7, 361-372. 
Conrad, O., Bechtel, B., Bock, M., Dietrich, H., Fischer, E., Gerlitz, L. et al. (2015). System for Automated Geoscientific Analyses (SAGA) v. 2.1.4. Geoscientific Model Development, 8, 1991-2007.

Darriba, D., Taboada, G.L., Doallo, R. \& Posada, D. (2012). jModelTest 2: more models, new heuristics and parallel computing. Nature Methods, 9, 772.

del Arco Aguilar, M.-J., Wildpret de la Torre, W., Pérez de la Paz, P.L., Rodríguez Delgado, O., Acebes Ginovés, J.R., García Gallo, A. et al. (2006). Mapa de vegetación de Canarias. GRAFCAN, Santa Cruz de Tenerife.

Emerson, B.C., Casquet, J., López, H., Cardoso, P., Borges, P.A.V., Mollaret, N. et al. (2017). A combined field survey and molecular identification protocol for comparing forest arthropod biodiversity across spatial scales. Molecular Ecology Resources, 17, 694-707.

Emerson, B.C., Oromí, P. \& Hewitt, G.M. (2000). Tracking colonisation and diversification of insect lineages on islands: MtDNA phylogeography of Tarphius canariensis (Coleoptera: Colydiidae) on the Canary Islands. Proceedings of the Royal Society of London B, 267, 21992205.

Fernández-Palacios, J.M., Arévalo, J.R., Balguerías, E., Barone, R., de Nascimento, L., Delgado, J.D. et al. (2017). La Laurisilva. Canaria, Madeira y Azores. Macaronesia Editorial, Santa Cruz de Tenerife.

Fernández-Palacios, J.M., Rijsdijk, K.F., Norder, S.J., Otto, R., de Nascimento, L., Fernández-Lugo, S. et al. (2016). Toward a glacial-sensitive model of island biogeography. Global Ecology and Biogeography, 25, 817-830.

Frichot, E., François, O. \& O'Meara, B. (2015). LEA: An R package for landscape and ecological association studies. Methods in Ecology and Evolution, 6, 925-929.

Frichot, E., Mathieu, F., Trouillon, T., Bouchard, G. \& Francois, O. (2014). Fast and efficient estimation of individual ancestry coefficients. Genetics, 196, 973-983.

García-Olivares, V., López, H., Patiño, J., Alvarez, N., Machado, A., Carracedo, J.C. et al. (2017). Evidence for mega-landslides as drivers of island colonisation. Journal of Biogeography, 44, 1053-1064.

García-Olivares, V., Patiño, J., Overcast, I., Salces-Castellano, A., López de Heredia, U., MoraMárquez, F. et al. (2019). A topoclimate model for Quaternary insular speciation. Journal of Biogeograpy, in press.

Goodman, K.R., Welter, S.C. \& Roderick, G.K. (2012). Genetic divergence is decoupled from ecological diversification in the Hawaiian Nesosydne planthoppers. Evolution, 66, 2798-2814.

Hardy, O.J. \& Vekemans, X. (2002). SPAGeDi: a versatile computer program to analyse spatial genetic structure at the individual or population levels. Molecular Ecology Notes, 2, 618-620. 
Hein, J., Schierup, M.H. \& Wiuf, C. (2005). Gene genealogies, variation and evolution. Oxford University Press, New York.

Hendrich, L., Morinière, J., Haszprunar, G., Hebert, P.D.N., Hausman, A., Köhler, F. et al. (2015). A comprehensive DNA barcode database for Central Europen beetles with a focus on Germany: adding more than 3500 identified species to BOLD. Molecular Ecology Resources, $15,795-818$.

Ho, S.Y.W. \& Duchêne, S. (2014). Molecular-clock methods for estimating evolutionary rates and timescales. Molecular Ecology, 23, 5947-5965.

Huson, D.H. \& Bryant, D. (2006). Application of phylogenetic networks in evolutionary studies. Molecular Biology and Evolution, 23, 254-267.

Jombart, T. \& Ahmed, I. (2011). adegenet 1.3-1: new tools for the analysis of genome-wide SNP data. Bioinformatics, 27, 3070-3071.

Karger, D.N., Conrad, O., Böhner, J., Kawohl, T., Kerft, H., Soria-Auza, R.W. et al. (2017). Climatologies at high resolution for the earth's land surface areas. Scientific Data, 4, 170122.

Kisel, Y. \& Barraclough, T.G. (2010). Speciation has a spatial scale that depends in levels of gene flow. The American Naturalist, 175, 316-334.

Kivimäki, I., Shimbo, M. \& Saerens, M. (2014). Developments in the theory of randomized shortest paths with a comparison of graph node distances. Physica A: Statistical Mechanics and its Applications 393, 600-616.

Lim, J.Y. \& Marshall, C.R. (2017). The true tempo of evolutionary radiation and decline revealed on the Hawaiian archipelago. Nature, 543, 710-713.

Lister, B.C. \& Garcia, A. (2018). Climate-driven declines in arthropod abundance restructure a rainforest food web. Proceedings of the National Academy of Sciences of the United States of America, 115, E10397-E10406.

Losos, J.B. \& Ricklefs, R.E. (2009). Adaptation and diversification on islands. Nature, 457, 830-836.

Machado, A., Rodríguez-Expósito, E., López, M. \& Hernández, M. (2017). Phylogenetic analysis of the genus Laparocerus, with comments on colonisation and diversification in Macaronesia (Coleoptera, Curculionidae, Entiminae). Zookeys, 651, 1-77.

Manolopoulou, I., Legarreta, L., Emerson, B.C., Brooks, S.D. \& Tavaré, S. (2011). A Bayesian approach to phylogeographic clustering. Interface Focus, 1, 909-921.

Marzol, V.M. (2008). Temporal characteristics and fog water collection during summer in Tenerife (Canary Islands, Spain). Atmospheric Research, 87, 352-361.

Mayr, E. (1942). Systematics and the origin of species from the viewpoint of a zoologist. Harvard University Press, Cambridge, Massachusetts. 
McRae, B.H., Dickson, B.G., Keitt, T.H. \& Shah, V.B. (2008). Using circuit theory to model connectivity in ecology, evolution, and conservation. Ecology, 89, 2712-2724.

McRae, B.H. \& Shah, V.B. (2009). Cicuitscape user's guide. Available at: www.circuitscape.org.

Murtagh, F. \& Legendre, P. (2014). Ward's Hierarchical Agglomerative Clustering Method: Which Algorithms Implement Ward's Criterion? Journal of Classification, 31, 274-295.

Papadopoulou, A., Anastasiou, I. \& Vogler, A.P. (2010). Revisiting the insect mitochondrial molecular clock: the mid-Aegean trench calibration. Molecular Biology and Evolution, 27, 1659-1672.

Patiño, J., Mateo, R.G., Zanatta, F., Marquet, A., Aranda, S.C., Borges, P.A.V. et al. (2016). Climate threat on the macaronesian endemic bryophyte flora. Scientific Reports, 6, 29156.

Peterman, W.E. (2018). ResistanceGA: An R package for the optimisation of resistance surfaces using genetic algorithms. Methods in Ecology and Evolution, 9, 1638-1647.

Peterman, W.E., Connette, G.M., Keitt, T.H. \& Shah, V.B. (2014). Ecological resistance surfaces predict fine-scale genetic differentiation in a terrestrial woodland salamander. Molecular Ecology, 23, 2402-2413.

Petkova, D., Novembre, J. \& Stephens, M. (2015). Visualising spatial population structure with estimated effective migration surfaces. Nature Genetics, 48, 94-100.

Phillips, S.J., Anderson, R.P. \& Schapire, R.E. (2006). Maximum entropy modeling of species geographic distributions. Ecological Modelling, 190, 231-259.

Rijsdijk, K.F., Hengl, T., Norder, S.J., Otto, R., Emerson, B.C., Ávila, S.P. et al. (2014). Quantifying surface-area changes of volcanic islands driven by Pleistocene sea-level cycles: biogeographical implications for the Macaronesian archipelagos. Journal of Biogeography, $41,1242-1254$.

Robinet, C. \& Roques, A. (2010). Direct implacts of recent climate warming on insect populations. Integratove Zoology, 5, 132-142.

Robinson, N., Regetz, J. \& Guralnick, R.P. (2014). EarthEnv-DEM90: A nearly-global, void-free, multi-scale smoothed, 90m digital elevation model from fused ASTER and SRTM data. ISPRS Journal of Photogrammetry and Remote Sensing, 87, 57-67.

Rominger, A.J., Goodman, K.R., Lim, J.Y., Armstrong, E.E., Becking, L.E., Bennett, G.M. et al. (2016). Community assembly on isolated islands: macroecology meets evolution. Global Ecology and Biogeography, 25, 769-780.

Rosindell, J. \& Harmon, L.J. (2013). A unified model of species immigration, extinction and abundance on islands. Journal of Biogeography, 40, 1107-1118. 
Schütte, A. \& Stüben, P.E. (2015). Molecular systematics and morphological identification of the cryptic species of the genus Acalles Schoenherr, 1825, with descriptions of new species (Coleoptera: Curculionidae: Cryptorhynchinae). Zootaxa, 3945, 001-051.

Shaw, K.L. \& Gillespie, R.G. (2016). Comparative phylogeography of oceanic archipelagos: Hotspots for inferences of evolutionary process. Proceedings of the National Academy of Sciences of the United States of America, 113, 7986-7993.

Sperling, F.N., Washington, R. \& Whittaker, R.J. (2004). Future climate change of the subtropical North Atlantic: implications for the cloud forests of Tenerife. Climate Change, 65, 103-123.

Stüben, P.E. \& Astrin, J.J. (2010). Molecular phylogeny in endemic weevils: revision of the genera of Macaronesian Cryptorhynchinae (Coleoptera: Curculionidae). Zoological Journal of the Linnean Society, 40-87.

Thirlwall, M.F., Singer, B.S. \& Marriner, G.F. (2000). 39Ar-40Ar ages and geochemistry of the basaltic shield stage of Tenerife, Canary Islands, Spain. Journal of Volcanology and Geothermal Research, 103, 247-297.

van Etten, J.R. (2017). R Package gdistance: Distances and Routes on Geographical Grids. Journal of Statistical Software, 76.

Vandergast, A.G., Gillespie, R.G. \& Roderick, G.K. (2004). Influence of volcanic activity on the population genetic structure of Hawaiian Tetragnatha spiders: fragmentation, rapid population growth and the potential for accelerated evolution. Molecular Ecology, 13, 17291743.

Walter, T.R., Troll, V.R., Cailleau, B., Belousov, A., Schmincke, H.-U., Amelung, F. et al. (2005). Rift zone reorganisation through flank instability in ocean island volcanoes: an example from Tenerife, Canary Islands. Bulletin of Volcanology, 67, 281-291.

Weigelt, P., Steinbauer, M.J., Cabral, J.S. \& Kreft, H. (2016). Late Quaternary climate change shapes island biodiversity. Nature, 532, 99-102.

Whittaker, R.J., Triantis, K.A. \& Ladle, R.J. (2008). A general dynamic theory of oceanic island biogeography. Journal of Biogeography, 35, 977-994.

Wiens, J.J. \& Graham, C.H. (2005). Niche conservatism: Integrating Evolution, Ecology, and Conservation Biology. Annual Review of Ecology, Evolution and Systematics, 36. 
Competing interests. The authors declare no competing interests.

Code availability. The $\mathrm{R}$ script for the delimitation of presumed biological species is available from GitHub: https://github.com/[to be made available upon acceptance]. 


\section{Canary Islands}
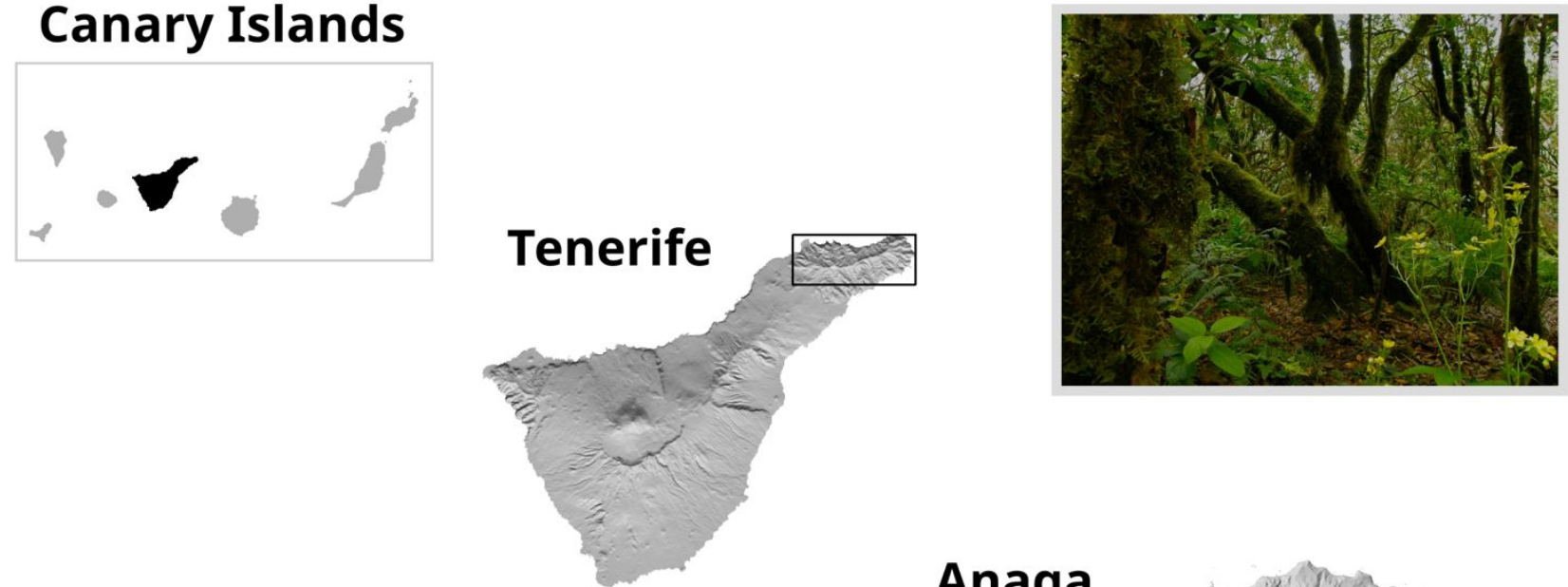

\section{Tenerife}

Anaga

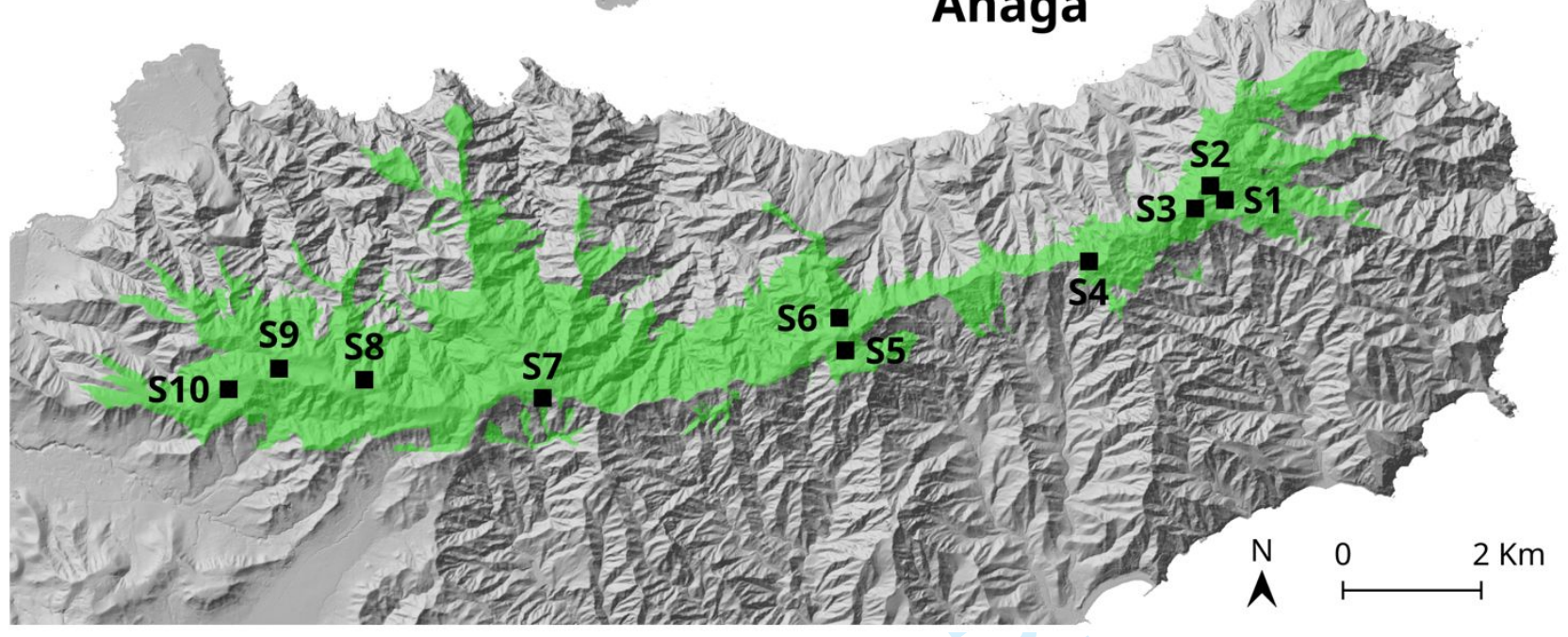

Figure 1 Sampling plots within the laurel forest of the Anaga peninsula of Tenerife, Canary Islands. The ten sampling plots are labelled, from east to west, S1 - S10, and the potential extent of the laurel forest within the Anaga peninsula (del Arco Aguilar et al. 2006) is shown in green. Top right inset shows typical laurel forest vegetation. 
Species (146)

(a) $51525354 \quad 55 \$ 665758 \quad 59510$

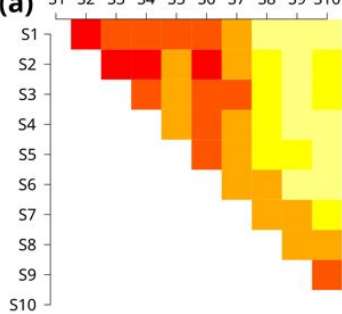

(b) $\bigcirc$ East $\bigcirc$ west

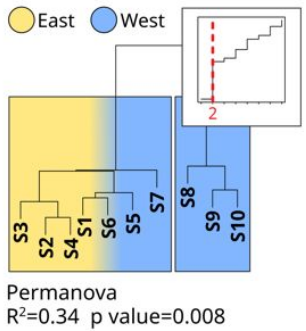

Phylogroups (105)
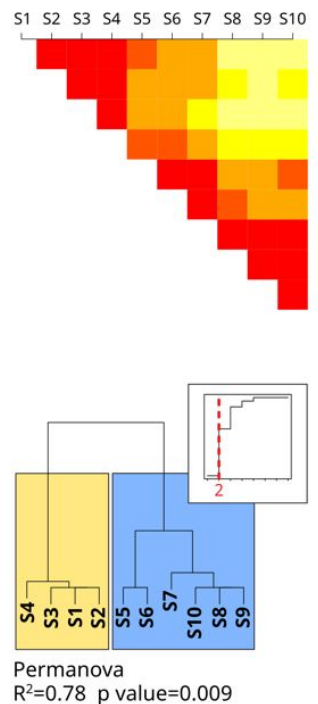

$\mathrm{N}_{\mathrm{ST}}(\mathbf{1 0 0 )}$
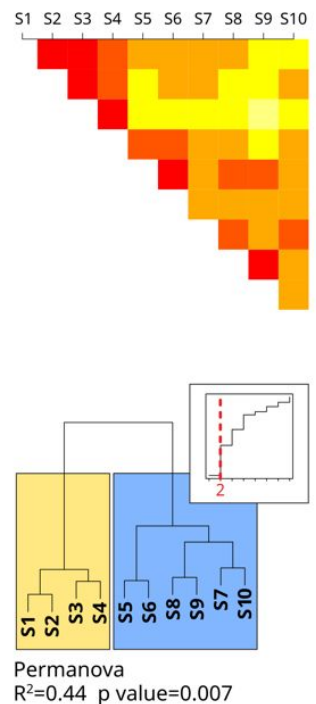

$\mathrm{G}_{\mathrm{ST}}(\mathbf{1 0 0 )}$
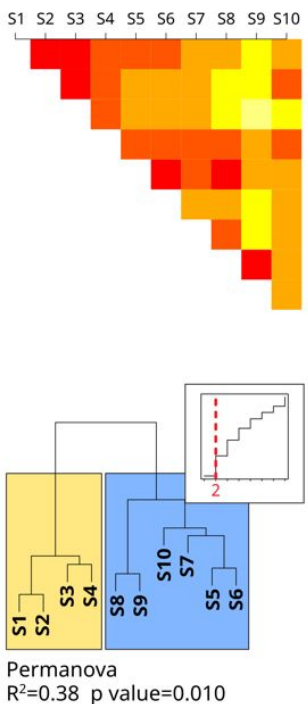

Haplotypes (950)
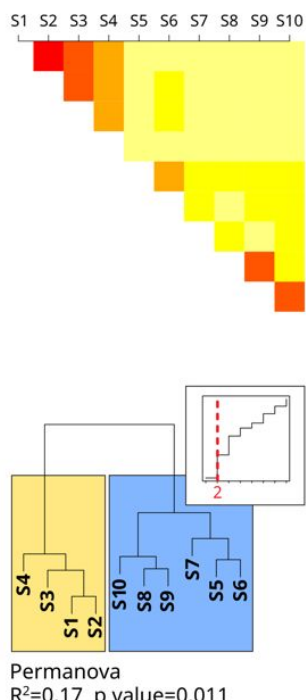

(c)

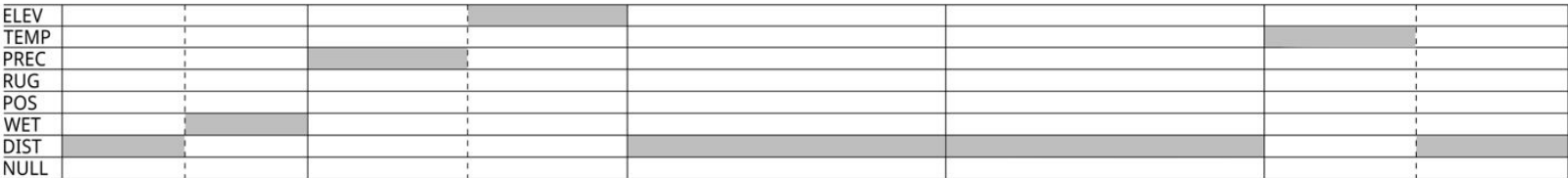

Figure 2 Patterns of community similarity among sampling plots. (a) Dissimilarity matrices among the ten sampling plots are presented at the level of presumed biological species (PBS) and four different treatments of intraspecific genetic variation: phylogroups, average $\mathrm{N}_{\mathrm{ST}}$ and $\mathrm{G}_{\mathrm{ST}}$ across all PBS, and all haplotypes across all PBS, where red represents maximum similarity between plots and light yellow represents maximum dissimilarity. Numbers in brackets correspond to the number of PBS sampled for each analysis, with the exception of haplotypes, where it represents the total number of haplotypes analysed. Sampling sites S1-S10 correspond to Fig. 1. (b) Hierarchical cluster analyses of dissimilarity matrices in $A$, with inset showing the increase in the average within-group pairwise similarity (y axis = homogeneity) across the different number of groups of plots defined by the hierarchical classification ( $\mathrm{x}$ axis). Repeated clustering of the four eastern and six western plots for the four treatments of intraspecific genetic variation, together with maximum increase in homogeneity, is highlighted with yellow and blue colours respectively. Significant differences 
between the four eastern vs the six western plots defined for each of the four dissimilarity matrices of intraspecific genetic variation were revealed by Permanova analyses. (c) Landscape resistance analyses showing optimal models (indicated with shading, separated by a dashed line when there is more than one optimal model) that explain genetic structure within each of the dissimilarity matrices in $A . \mathrm{ELEV}=$ elevation, $\mathrm{TEMP}=$ temperature, $\mathrm{PREC}=$ precipitation, $\mathrm{RUG}=$ topographic rugosity, $\mathrm{POS}=$ topographic position index, WET $=$ topographic wetness index, DIST $=$ Euclidean distance, NULL $=$ null model. See Table S3 for specific details. 
(a)

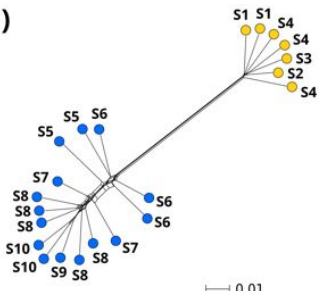

(b)

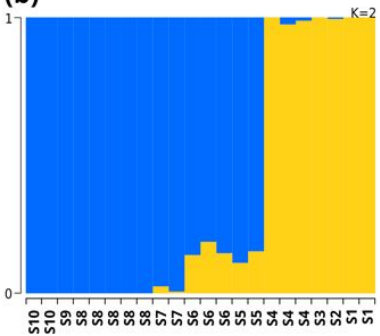

Laparocerus subnodosus

(a)

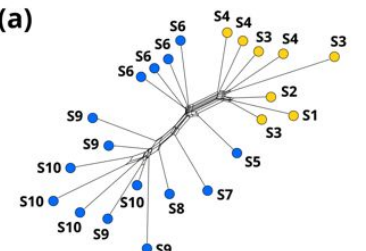

$\longmapsto 0.01$

(b)

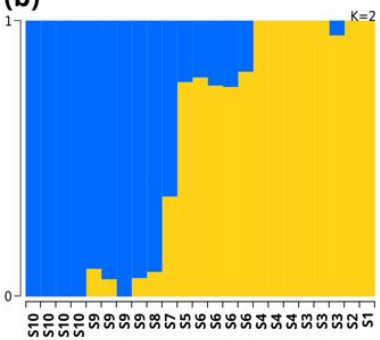

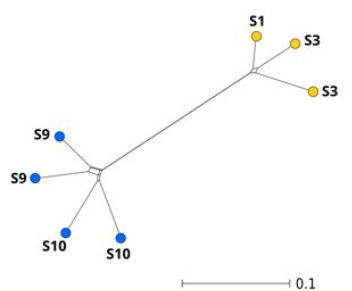
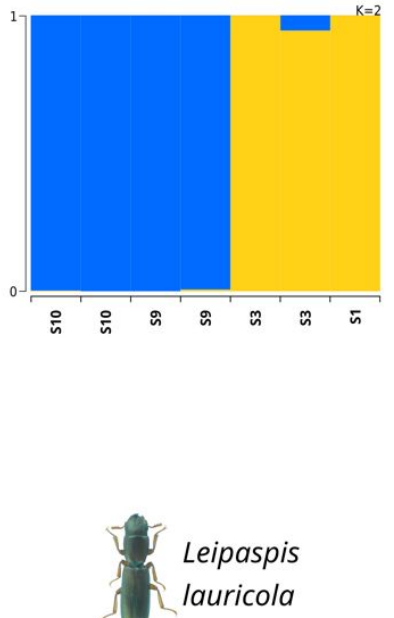

lauricola
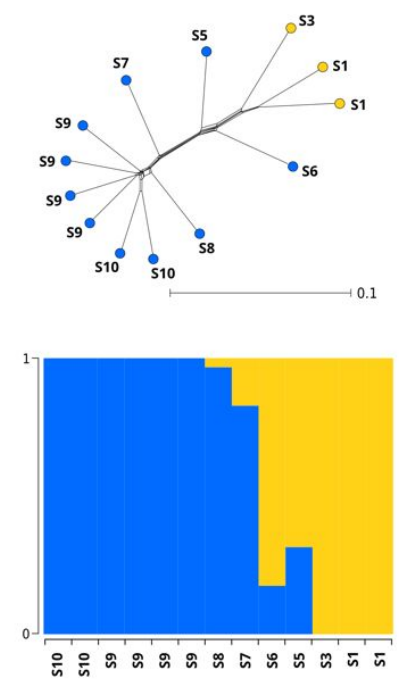
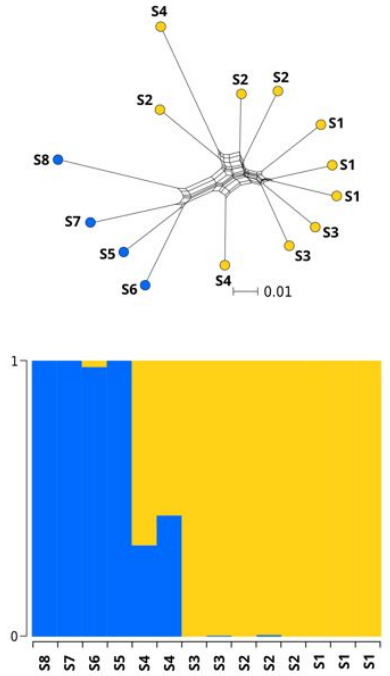

Paradromius amoenus
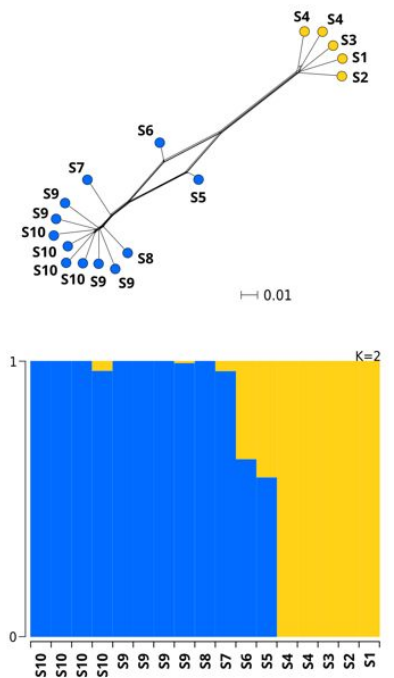
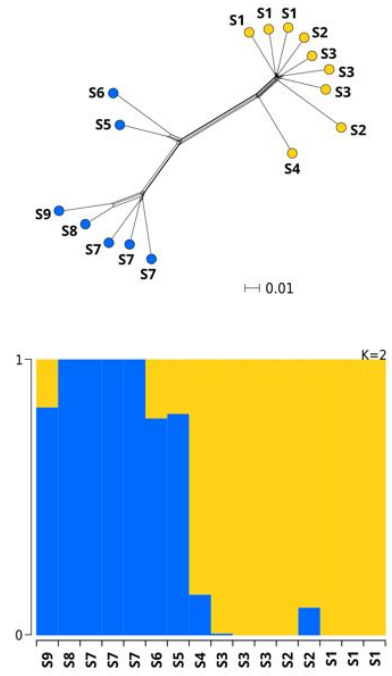

Rhopalomesites persimilis
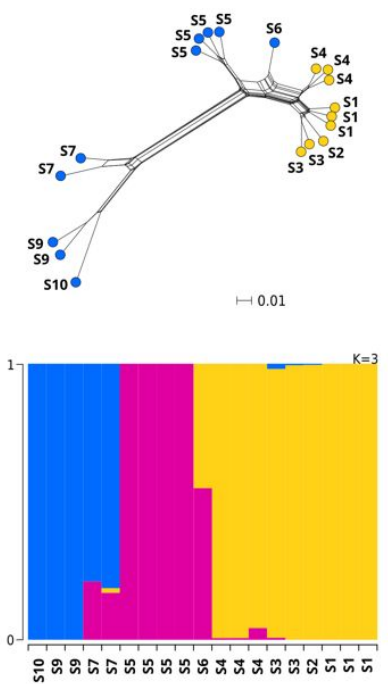

Figure 3 Regional structuring of beetle genetic variation across the Anaga peninsula of Tenerife. Based on East-West regional patterns of mtDNA structure, ddRAD-seq data was generated for eight PBS (Table S1). (a) Haplotype networks constructed with ddRAD-seq data using the NeighborNet method in SplitsTree. The four eastern and six western plots are highlighted with yellow and blue 
colours, respectively, and codes correspond to sampling locations presented in Fig. 1. Each circle represents an individual, scale bars represent the proportion of inferred mutational change along branches. (b) Ancestry estimation matrices from model-based clustering analyses, using ddRADseq data. When optimal K (number of inferred ancestral populations) was inferred to be two or three, this is indicated with $\mathrm{K}=2$ or $\mathrm{K}=3$ respectively. $\mathrm{K}$ was forced to two for Cardiophorus sp. and Leipaspis lauricola, where optimal $\mathrm{K}$ was inferred to be one, to test the specific hypothesis of regional structuring between eastern and western populations. Blue and yellow represent proportion of genomic assignment to eastern and western ancestry respectively. In the case of $R$. persimilis, a third (central) ancestral population is represented in pink. See Fig. S1 for mtDNA haplotype networks and ddRAD-seq PCA plots for each PBS, and all results for eight PBS with no regional structuring of mtDNA. 

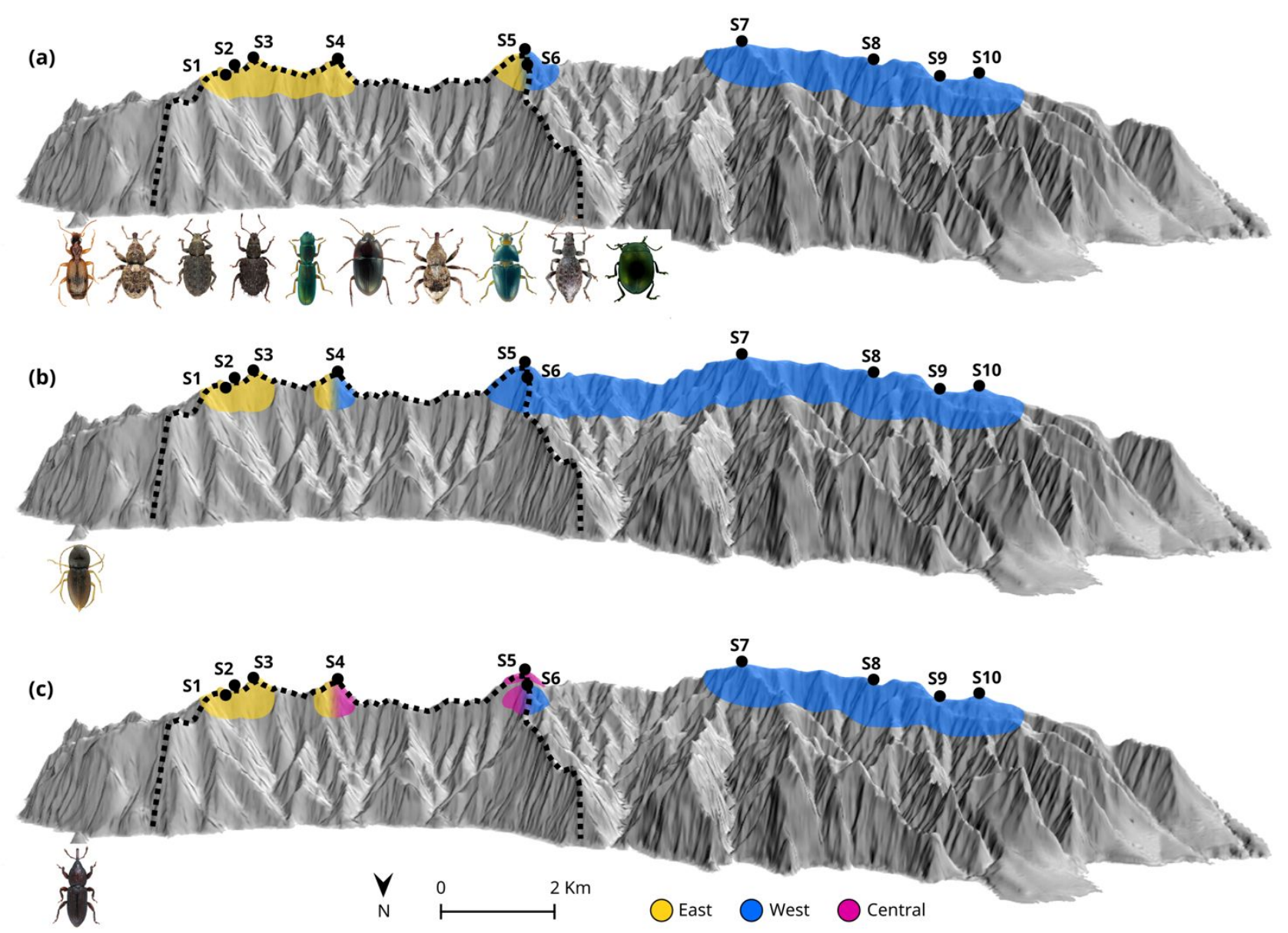

Figure 4 Repeated regional structuring of beetle intraspecific nuclear genomic variation across the Anaga peninsula. The Anaga peninsula is shown looking down onto its northern flank, with sampling sites labelled as in Fig. 1. Broken black lines denote the limits of a mega-landslide estimated to have occurred between 4.1 - 4.7 Ma (Walter et al. 2005). Different ancestral populations inferred from individual ancestry coefficients estimated with the program sNMF (see Methods) are represented with different colours. Twelve of sixteen species of beetle analysed with ddRAD-seq data (see Fig. S1) present evidence for regional structuring of inferred ancestral gene pools with geographically intermediate admixture, each fitting one of three models: (a) eastern (yellow) and western (blue) populations and admixed genomes centred around S5 and S6. This model is observed for the ten 
species pictured, from left to right, Paradromius amoenus, Acalles globulipennis, Laparocerus subnodosus, Laparocerus impressicollis, Leipaspis lauricola, Amaroschema gaudini, Silvacalles instabilis, Xestus throscoides, Laparocerus ellipticus and Chrysolina costalis; (b) Cardiophorus sp. (pictured) is separated into eastern (yellow) and western (blue) populations, distributed either side of admixed genomes in S4; (c) Rhopalomesites persimilis (pictured) is separated into eastern (yellow), central (pink) and western (blue) populations, with admixed genomes in geographically intermediate plots between east and central (S4) and central and west (S6). 